\title{
SSC12-I-2
}

\section{Nanosatellites for Earth Environmental Monitoring: The MicroMAS Project}

\author{
William J. Blackwell, G. Allen, S. Conrad, C. Galbraith, R. Kingsbury, R. Leslie, P. McKinley, I. Osaretin, W. \\ Osborn, B. Reid, L. Retherford, M. Scarito, C. Semisch, M. Shields, M. Silver, D. Toher, R. Wezalis, K. Wight \\ Lincoln Laboratory, Massachusetts Institute of Technology \\ 244 Wood St., Lexington, MA 02420-9185 U.S.A; 781-981-7973 \\ wjb@LL.MIT.EDU
}

\author{
Kerri Cahoy, David W. Miller, Anne Marinan, Sung Wook Paek, Eric Peters, Frank Hall Schmidt, Bruno Alvisio, \\ Evan Wise, Rebecca Masterson, Danilo Franzim Miranda \\ Space Systems Laboratory, Massachusetts Institute of Technology \\ 77 Massachusetts Ave., Cambridge, MA 02139 \\ kcahoy@mit.edu \\ Neal Erickson \\ Department of Radio Astronomy \\ University of Massachusetts \\ Amherst, MA 01003
}

\begin{abstract}
The Micro-sized Microwave Atmospheric Satellite (MicroMAS) is a dual-spinning 3U CubeSat equipped with a passive microwave spectrometer that observes nine channels near the 118.75-GHz oxygen absorption line. The focus of this MicroMAS mission (hereafter, MicroMAS-1) is to observe convective thunderstorms, tropical cyclones, and hurricanes from a near-equatorial orbit. The MicroMAS-1 flight unit is currently being developed by MIT Lincoln Laboratory, the MIT Space Systems Laboratory, and the MIT Department of Earth and Planetary Sciences for a 2014 launch to be provided by the NASA CubeSat Launch Initiative program. As a low cost platform, MicroMAS offers the potential to deploy multiple satellites than can provide near-continuous views of severe weather. The existing architecture of few, high-cost platforms, infrequently view the same earth area which can miss rapid changes in the strength and direction of evolving storms thus degrading forecast accuracy. The 3U CubeSat has dimensions of $10 \times 10 \times 34.05 \mathrm{~cm}^{3}$ and a mass of approximately $4 \mathrm{~kg}$. The payload is housed in the "lower" $1 \mathrm{U}$ of the dualspinning 3U CubeSat, and is mechanically rotated approximately once per second as the spacecraft orbits the Earth. The resulting cross-track scanned beam has a FWHM beam width of $2.4^{\circ}$, and has an approximately 20 -km diameter footprint at nadir incidence from a nominal altitude of $500 \mathrm{~km}$. Radiometric calibration is carried out using observations of cold space, the Earth's limb, and an internal noise diode that is weakly coupled through the RF front-end electronics. In addition to the dual-spinning CubeSat, a key technology development is the ultra-compact intermediate frequency processor (IFP) module for channelization, detection, and analog-to-digital conversion. The payload antenna system and RF front-end electronics are highly integrated, miniaturized, and optimized for low-power operation. To support the spinning radiometer payload, the structures subsystem incorporates a brushless DC zerocogging motor, an optical encoder and disk, a slip ring, and a motor controller. The attitude determination and control system (ADCS) utilizes reaction wheels, magnetorquers, Earth horizon sensors, peak power tracking, a magnetometer, and a gyroscope. The communications system operates at S-band using the Open System of Agile Ground Stations (OSAGS) with a 2.025-2.120 GHz uplink and 2.200-2.300 GHz downlink at 230 kbps. MicroMAS-1 uses a Pumpkin CubeSat Motherboard with a Microchip PIC24 microcontroller as the flight computer running Pumpkin’s Salvo Real Time Operating System. Thermal management includes monitoring with thermistors, heating, and passive cooling. Power is generated using four double-sided deployable $3 \mathrm{U}$ solar panels and one $2 \mathrm{U}$ bodymounted panel with UTJ cells and an electrical power system (EPS) with $30 \mathrm{~W}$-hr lithium polymer batteries from Clyde Space. Tests with the MicroMAS-1 Engineering Design Model (EDM) have resulted in modifications to the spinning assembly, stack and ADCS system and have informed the development of the flight model subsystems.
\end{abstract}

\section{INTRODUCTION}

Passive microwave observations from space have proven to be a valuable measurement type for assimilation into numerical weather prediction models. The cloud penetrating capability afforded by microwave provides allweather performance, whereas infrared observations can 
be obscured by even partial cloud cover ${ }^{1,2}$. The research team comprising MIT Lincoln Laboratory, the MIT Space Systems Laboratory (SSL), and the MIT Department of Earth and Planetary Sciences is building a dualspinning $3 \mathrm{U}$ CubeSat hosting a passive microwave radiometer to demonstrate the viability of low-cost, easilylaunchable nanosatellites for high-fidelity Earth Science research that would provide game-changing advances by reducing costs by at least an order of magnitude while increasing robustness to launch and sensor failures.

MicroMAS-1 will provide unique observations of hurricane dynamics and demonstrate a new, transformative satellite platform for future meteorological satellites that support operational numerical weather prediction models, climate records, and scientific investigations. The MicroMAS-1 flight unit consists of a $2 \mathrm{U}$ spacecraft bus with supporting subsystems and a $1 \mathrm{U}$ science payload containing a passive microwave spectrometer that is mechanically rotated as the spacecraft orbits the Earth. The MicroMAS-1 flight unit is currently being developed in anticipation of a 2014 launch through the NASA Educational Launch of Nanosatellites (ELaNa) program.

In this paper, we describe the science mission, satellite bus design, and results from testing done on an EDM of the satellite.

\section{SCIENCE OVERVIEW}

Weather forecasting and warning applications rely increasingly on integrated observations from a variety of systems that are asynchronous in time and are nonuniformly spaced geographically. Critical observing system features include rapid update and full volumetric coverage. On regional scales, the combination of satellite data with automated meteorological measurements from aircraft and with a network of ground-based radars and meteorological instruments reporting in real time has been shown to provide enhanced nowcasting and shortterm forecasting capabilities. Such capabilities improve severe local storm warnings (including forecasts of storm initiation, evolution, and decay), and they support activities such as construction, road travel, the needs of the aviation system (both civil and military), and recreation. The MicroMAS work focuses on improved rapidupdate capabilities provided by a low-earth-orbit satellite constellation.

Oxygen band channels measure blackbody radiation emanating from atmospheric layers which are many kilometers thick and are centered at altitudes ranging between the surface and the stratosphere, depending on the observed radio frequency. Both the $60-$ and $118-\mathrm{GHz}$ resonant bands of oxygen exhibit similar ranges of atmospheric transmittances and have corresponding altitude responses, while also having a usefully different response to hydrometeors. The larger particles at the top of a precipitating column reflect the cold radiance of space into the antenna beam, and the altitude of the reflecting layer can be inferred because each channel is sensitive to a unique vertical region of the atmosphere. Cell-top altitude retrievals using $118-\mathrm{GHz}$ oxygen band spectral images have been described ${ }^{7}$, where rms accuracies approaching $1 \mathrm{~km}$ were suggested; cell-top altitude is related in part to vertical winds and precipitation rates.

\section{Background}

Microwave atmospheric remote sensing systems observe the radiance from the atmosphere at microwave wavelengths. An antenna receives the radiation and then supporting electronics detects and amplifies this signal within a certain frequency band. This detected power level is used to generate temperature and moisture profiles of the atmosphere, which can inform meteorological modeling$^{2}$. Atmospheric elements have different absorption spectra, and sampling at different frequencies permits the observation of different atmospheric constituents which probe different depths of the atmosphere ${ }^{2}$. For example, the microwave molecular oxygen line is very good for determining temperature distribution because its behavior is stable and well-known ${ }^{3}$. Figure 1 shows atmospheric attenuation for various atmospheric species. MicroMAS- 1 will observe in bands near the $118.75-\mathrm{GHz}$ oxygen absorption line.

Fast-changing weather systems like cyclones and tropical storms require frequent coverage and quick data turnaround in order to adequately characterize their behavior ${ }^{4}$. This is a major limitation of current spacebased radiometer observing systems that will be addressed by MicroMAS-1 and follow-on missions.

The best current satellite spatial resolutions for routinely observing the warm cores of hurricanes, or "altitude slicing" their rain bands to determine altitudes, are $\sim 50 \mathrm{~km}$ on AMSU (Advanced Microwave Sounding Unit), 25 $\mathrm{km}$ on SSMIS (Special Sensor Microwave Imager Sounder) ${ }^{6}$, an $\sim 33 \mathrm{~km}$ on ATMS (Advanced Technology Microwave Sounder) ${ }^{5}$ launched in 2011. None of these satellites revisit any latitude lower than $50^{\circ}$ more than twice daily ( 12-hour intervals). MicroMAS-1, because of its low inclination orbit and low altitude, will not only revisit most hurricanes at least twice daily but sometimes may visit two or even three times daily at $\sim 100$-minute intervals for many storms in the outer $8^{\circ}$ latitude bands, thus revealing for the first time the character of rapid thermal variations of a large variety of storms. 


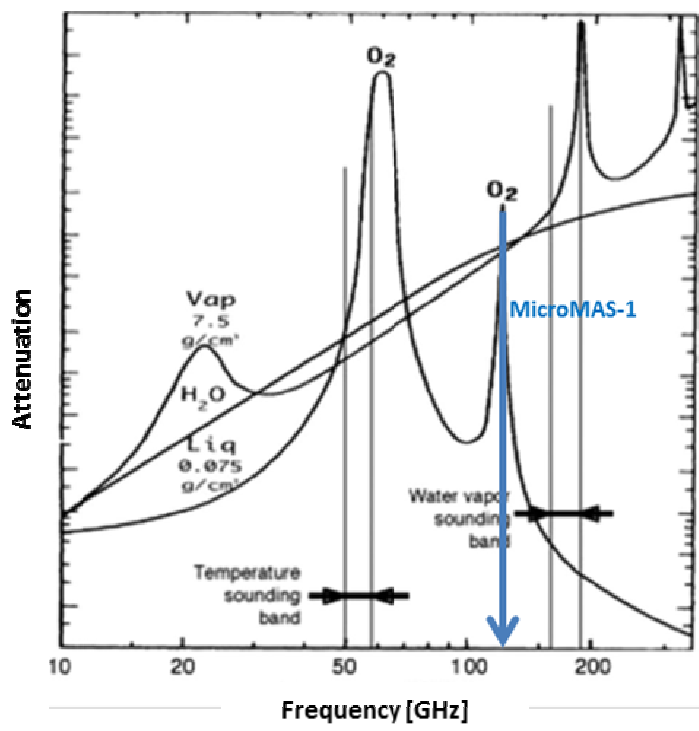

Figure 1: Microwave Absorption Spectrum ${ }^{3}$

Figure 2 shows sample images from an airborne microwave radiometer system of the eyewall of Cyclone Oliver in 1993. The data used to generate the images were $118 \mathrm{GHz}$ spectral data spaced 3 hours apart ${ }^{7}$. This is the frequency range of operation for MicroMAS-1, and we expect to be able to generate similar images with a shorter revisit time.
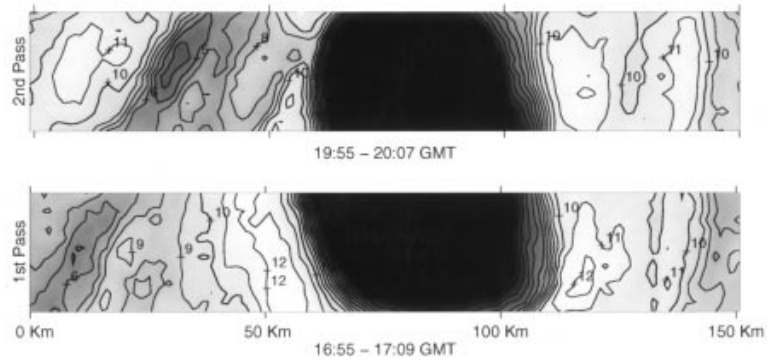

Figure 2: Image of cell-top altitudes from Cyclone Oliver generated from NASA ER-2 data ${ }^{7}$

\section{Goals}

The MicroMAS-1 project represents one of the first attempts to perform an operational remote sensing science mission based upon the standard CubeSat modular architecture. Current Earth-observing satellites designed to collect atmospheric profile data such as the Suomi National Polar Orbiting Partnership (NPP) ${ }^{8}$ or the Defense Meteorological Satellite Program (DMSP) ${ }^{9}$ can cost in the $\$ 100 \mathrm{M}-\$ 1 \mathrm{~B}+$ range and require 5-10 years to develop and launch. Although each successful flight vehicle demonstrates an impressive array of capabilities, many large space system acquisition programs are continuously plagued by cost and schedule overruns ${ }^{10}$. MicroMAS1 seeks to implement one specific data collection capa- bility on a much smaller scale. The MicroMAS-1 flight model promises comparable science return to other Earth observation satellite systems that are larger, heavier, require more power, and are more expensive to build and launch. In order to achieve this large reduction in cost and development effort, MicroMAS-1 is pursuing a strategy to design the system such that it fits within the 3U CubeSat standard and uses as many commercially available components as possible.

The main science goals of MicroMAS-1 are:

1. Collect millimeter-wave radiometric images of tropical storms, hurricanes and cyclones.

2. Decrease ground sample distance (GSD), which in this system is equivalent to ground-projected instantaneous field of view (GIFOV), to provide finer detail in storm characterization.

3. Increase observational frequency to better resolve rapid cyclone development and dynamics.

4. Increase the number of spectral bands to improve the vertical resolution of the atmospheric temperature profile.

MicroMAS-1 is especially relevant for the following reasons:

(i) Temperature and moisture sounding missions (like $\mathrm{PATH}^{3}$ and JPSS) would greatly benefit from improved performance, especially in the critical boundary layer and in the presence of clouds. Both of the proposed sensing systems for Precipitation and All-Weather Temperature and Humidity Mission (PATH) ${ }^{3}$, the Geostationary Synthetic Thinned Aperture Radiometer, GeoSTAR ${ }^{3}$, and the Geostationary Millimeter-wave Array Spectrometer (GeoMAS) ${ }^{11}$, could exploit distributed microwave CubeSat spacecraft operation for improved performance and calibration, especially in mid-to-high latitudes where geostationary sensing coverage and performance is relatively poor.

(ii) An important new science objective of the Surface Water Ocean Topography mission (SWOT) is to enable useful radar altimetry measurements in regions near the coast, which would benefit from high-spatial resolution wet-tropospheric path delay corrections near land that could be provided by a low-cost millimeter-wave microwave sounding fleet. Such retrievals would significantly improve SWOT performance by enabling wet tropospheric path delay retrievals with an rms error of less than $1 \mathrm{~cm}$ to within $3 \mathrm{~km}$ from the coast ${ }^{12}$.

(iii) Surface temperature retrievals are significantly improved by the frequent microwave observations that would be afforded from a fleet of CubeSat sensors, from over $2.5 \mathrm{~K} \mathrm{rms}$ to below $1 \mathrm{~K}$ rms, thus offering potential improvements to the Snow and Cold Land Processes 
(SCLP) mission ${ }^{11}$. Furthermore, retrieval of precipitation and cloud microphysical properties has been shown to benefit from frequent microwave observations, thus offering potential improvements to the passive microwave components of the Advanced Composition Explorer (ACE) and Global Precipitation (GPM) missions ${ }^{11}$. GPM is a NASA mission, scheduled to launch in February 2014 at an altitude of $\sim 500 \mathrm{~km}$ and inclination of about $65^{\circ}$.

\section{Payload and Radiometer Miniaturization}

The microwave sensor is a total-power radiometer/spectrometer based on Monolithic Microwave Integrated Circuit (MMIC) technology. The basic system architecture is identical to several on-orbit systems (AMSU, Aquarius, Microwave Limb Sounder, etc.) ${ }^{13}$. The principal challenge of developing a radiometer payload for a CubeSat is the high level of integration necessary to meet size, weight, and power (SWaP) requirements.

The payload will use a diffraction-limited 9-cm aperture antenna with a $3 \mathrm{~dB}$ beam width of $2.4^{\circ}$ and nadir resolution of $\sim 20 \mathrm{~km}$ to observe nine channels on the wings of a strong oxygen resonance at $118.75 \mathrm{GHz}$. The channelization is based on previous work ${ }^{11}$, with typical channel bandwidths of $625 \mathrm{MHz}$ and typical NEDTs of approximately $0.3 \mathrm{~K}$. A surface-sensitive channel is measured near $108 \mathrm{GHz}$. Together, these observations result in the measurement of temperature and precipitation structures at altitudes from the surface to $20 \mathrm{~km}$. The spinner assembly rate of $60 \mathrm{rpm}(\sim 1 \mathrm{~Hz})$ to track across the Earth permits Nyquist sampling and the opportunity to improve spatial resolution after performing ground-based image sharpening.

A single receiver will be developed to cover 108-119 $\mathrm{GHz}$, based partly on the UMass-Amherst development of the SEQUOIA focal plane array $(85-116 \mathrm{GHz})$ for the Large Millimeter Telescope. The radiometer will be built in two parts: 1) a noise source and preamplifier, and 2) a module with additional gain at $118 \mathrm{GHz}$, a mixer, and an IF amplifier. The local oscillator (LO) for the mixer will be at $90 \mathrm{GHz}$, produced by a tripler housed in a separate block. The RF gain will exceed $40 \mathrm{~dB}$ and the IF gain will exceed $30 \mathrm{~dB}$. Frontend receiver noise figure is not expected to exceed $5 \mathrm{~dB}$. Total power consumption of all $118-\mathrm{GHz}$ receiver components is not expected to exceed $2 \mathrm{~W}$ and total mass is not expected to exceed $500 \mathrm{~g}$. An ultra-compact intermediate frequency processor module for channelization, detection, and analog to digital (A/D) conversion is a key technology development for MicroMAS-1. The antenna system and radio-frequency (RF) front-end electronics are highly integrated and miniaturized.

\section{Future Applications}

The relatively low cost of CubeSat remote sensing to be demonstrated by MicroMAS-1 facilitates the deployment of a constellation of sensors, spaced equally around several orbit planes. For example, a dozen satellites could provide average global revisit times approaching 20 minutes, a revolutionary step forward for atmospheric sounding and precipitation science. A small fleet of MicroMAS Satellites could yield high-resolution global temperature and water vapor profiles, as well as cloud microphysical and precipitation parameters. Furthermore, the recent announcement of the NASA CubeSat initiative to support spacecraft up to $6 \mathrm{U}^{14}$ enables much more sophisticated sensing systems with higher vertical and spatial resolution.

\section{MISSION OVERVIEW}

\section{Concept of Operations}

MicroMAS-1 will be launched through NASA's ELaNa program in 2014. The desired orbit has an inclination between 20 and $30^{\circ}$ and initial altitude between 475 and $600 \mathrm{~km}$, although other orbit inclinations could be tolerated. Once separated from the launch vehicle, the satellite will deploy solar panels and establish a communications link with the ground station before orienting to a stable, nadir-pointing attitude configuration as shown in Figure 3. While keeping the $2 \mathrm{U}$ main bus nadirpointing, MicroMAS-1 will then spin up the $1 \mathrm{U}$ payload module so the radiometer field of view sweeps across the ground track at a rate of approximately $1 \mathrm{~Hz}(60 \mathrm{rpm})$. After a nominal operational lifetime of one year, with no propulsion on board, the orbit will eventually decay until the spacecraft re-enters the atmosphere within the required 25 year time period ${ }^{15}$. Figure 3 shows MicroMAS-1 on orbit with solar panels deployed as well as the radiometer field of view.

\section{Payload Operation}

Throughout its one-year mission, MicroMAS-1 will collect data from the rotating radiometer payload. The payload rotation allows the radiometer to repeatedly view both cold space and the Earth's limb in order to be able to carry out radiometric calibration. Additional calibration will be provided by an on-board internal noise diode $^{8}$. The rotation of the payload directs a cross-track scanned beam with FWHM beam width of $2.4^{\circ}$, yielding an approximately $20 \mathrm{~km}$ diameter footprint from a nominal orbit altitude of $500 \mathrm{~km}$.

\section{BUS DESIGN}

MicroMAS-1 uses a modified CubeSat architecture. It features a unique dual-spinner assembly, 3-axis stability and pointing, deployable solar panels for increased power generation, and data management. 


\section{CubeSats}

A CubeSat is a picosatellite with strict standards for size, mass, power, and launch configurations. COTS components are an integral part of CubeSat design, and there are companies that specifically target the CubeSat market (e.g. Pumpkin and Clyde Space). Due to this standardization and availability of COTS components, CubeSats are relatively cheap and simple to integrate when compared with other nanosatellites and have space heritage. Each unit (U) of a CubeSat is a $10 \times 10 \times 10$ $\mathrm{cm}^{3}$ cube capable of supporting a mass of $1.33 \mathrm{~kg}^{9}$. The low cost and relative simplicity and availability of CubeSat compatible components are making these satellites increasingly popular, particularly in university and research settings.

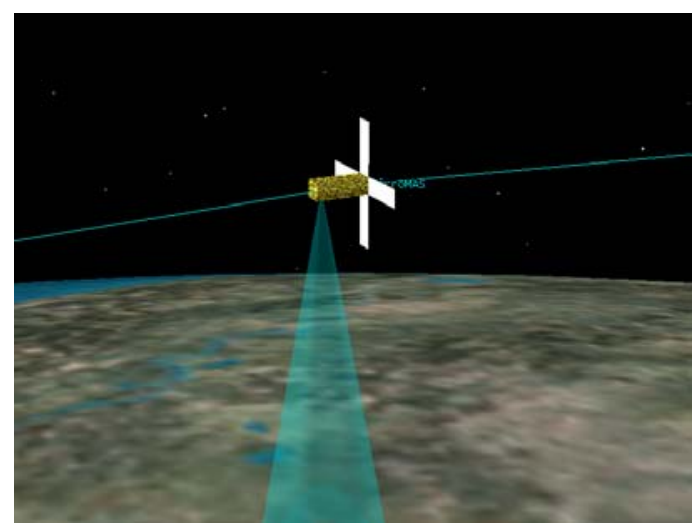

Figure 3: MicroMAS-1 Mission Configuration

MicroMAS-1, shown in Figure 4, is a dual-spinning 3U CubeSat $\left(10 \times 10 \times 34.05 \mathrm{~cm}^{3}\right)$ that consists of a $1 \mathrm{U}$ payload and supporting $2 \mathrm{U}$ bus connected via a spinner assembly. It operates in a dual-spin configuration with the payload spinning at a rate of $1 \mathrm{~Hz}(60 \mathrm{rpm})$ while the bus maintains a stable, nadir-pointing configuration.

The design of the $2 \mathrm{U}$ CubeSat bus is comprised of six main subsystems: structures, thermal, ADCS, power, and data management (communications and avionics).

\section{Structure}

The design for the flight model (FM) was greatly informed by the SWaP test model and EDM that were developed in the MIT Space Systems Laboratory during Summer 2011. The process of designing, building, and testing these models provided the team with valuable insights into component configuration, acceptable mounting methods, and the assembly process. Lessons learned from the SWaP model and EDM are captured in the Test Results section.

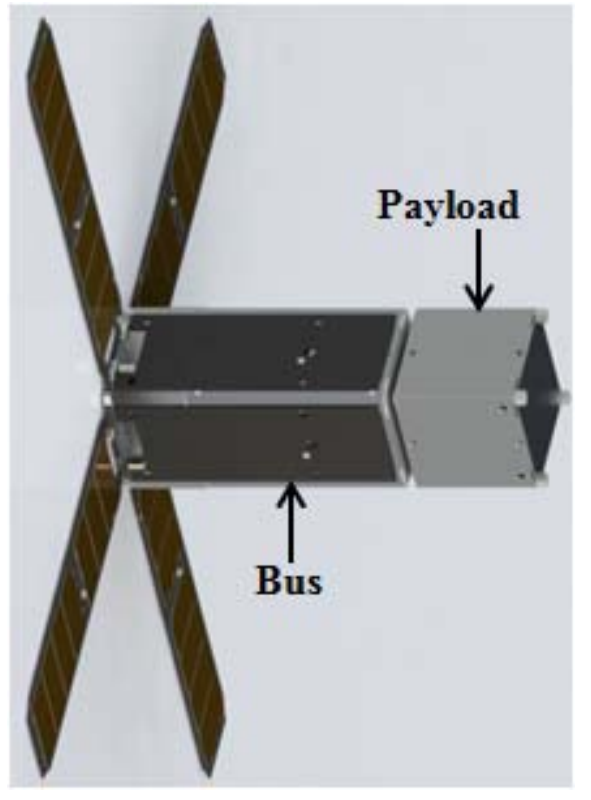

Figure 4: MicroMAS-1 CubeSat

The spacecraft bus is comprised of a $0.5 \mathrm{U}$ external ADCS unit and a custom-length $1.5 \mathrm{U}$ skeletonized chassis from Pumpkin Inc ${ }^{16}$. Together, the bus stack matches the length of a standard $2 \mathrm{U}$ CubeSat and leaves a full $1 \mathrm{U}$ for the payload, including a $6 \mathrm{~mm}$ gap between the two bus structures to allow for spinner assembly clearance. The internal components of the FM bus stack are arranged in the following order, from bottom to top per Figure 6: custom ADCS interface board; S-band radio board; battery; EPS; motherboard; custom interface board for motor controller and magnetometer; encoder head, electronics, and disk; and spinner assembly motor. The location of the motherboard is of particular importance, as it must line up with one of the three access ports in the P-POD for access to the battery charging port and remove-before-flight pin after integration with the launch vehicle ${ }^{17}$. A comparison of the internal components between the EDM and the FM can be seen in Figure 5 and Figure 6, respectively.

\section{Thermal}

Passive thermal management will be used as much as possible. Thermal tape will passively radiate heat out of the $2 \mathrm{U}$ bus structure. Currently, no satellite-level active control components are planned. However, individual satellite components will require active control for heating, especially components with limiting operational temperature ranges, such as the batteries. The thermal tape used to wrap the chassis has an absorptivity and emissivity of 0.5 except in the visible spectrum, where 
the absorptivity is 0.14 . With these values, the average flux into MicroMAS by the heater plate, which emits in the IR spectrum, was approximately $20 \mathrm{~W}$ The IR radiation emitted by earth will contribute a constant $4.25 \mathrm{~W}$. Solar Input will greatly vary depending on the orientation of MicroMAS to the sun vector. In the slimmest profile, MicroMAS will be showing $0.034 \mathrm{~m}^{2}$ to the sun, resulting in a solar flux of $6.5 \mathrm{~W}$ to the spacecraft. In a worst thermal scenario, where the deployed solar panels are pointed at the sun, the exposed are will be $0.146 \mathrm{~m}^{2}$ with an absorptivity of 0.9 for the solar panels. The average heat flux will be $91.5 \mathrm{~W}$. Also, albedo will account for an additional $3.1 \mathrm{~W}$ of heat flux while in the sun. At zero degrees Celsius, they are capable of radiating 21.4 $\mathrm{W}$ apiece. MicroMAS will have an average heat flux of $68 \mathrm{~W}$ over the course of the orbit. Adding in avionics, the total heat load will be about $84 \mathrm{~W}$. With four panels radiating $21.4 \mathrm{~W}$, and not including bus radiation, MicroMAS should actually operate below $0{ }^{\circ} \mathrm{C}$.

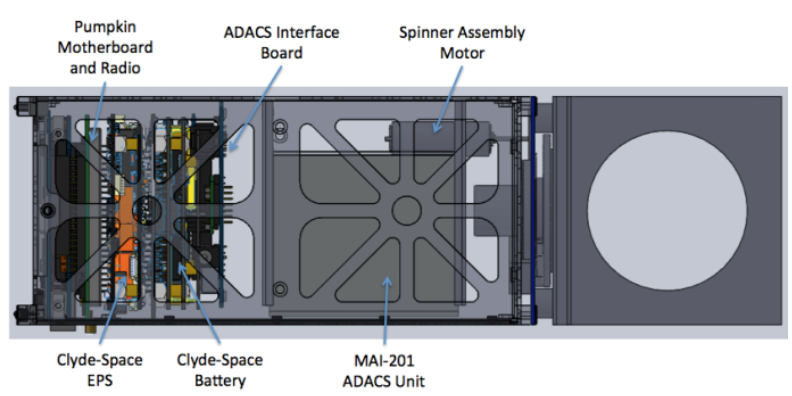

Figure 5: MicroMAS-1 Engineering Development Model internal components

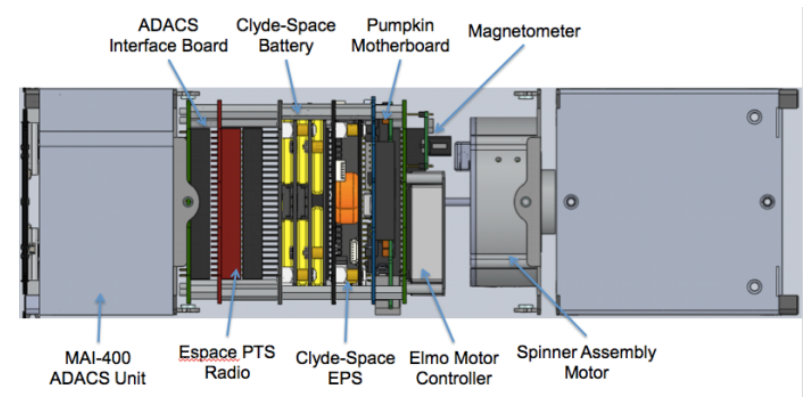

Figure 6: MicroMAS-1 Flight Model internal components

\section{ADCS}

The original design for MicroMAS-1 required the entire spacecraft to rotate about its long axis. While spinstabilized satellites have been around for many years, gyroscopic stiffness makes it difficult to maintain nadir pointing. A nadir-pointing spacecraft needs to rotate about its orbit-normal axis once per orbit (in LEO, this would be a period of roughly 90 minutes). Exchanging momentum while precessing in such an orbit requires high torques that are not readily available with COTS ADCS solutions. The radiometer pointing requirements (30 arcminutes pointing accuracy and 6 arcminutes attitude determination accuracy) requires actuators capable of fine manipulation of the spacecraft's angular momentum, such as reaction wheels. Additional control issues arise when considering that the ADCS system rotates with the spacecraft, so transferring angular momentum from one off-axis reaction wheel to the other off-axis reaction wheel as the spacecraft spins would require significant coordination between the sensors providing angular rate and displacement data and the reaction wheel controllers, thus requiring more power and more processor time on the spacecraft's flight computer.

Rather than spinning the entire spacecraft, then, a dualspinning satellite design offers a solution. It is important to note that application of a counter-mass system would also provide a good solution, however the weight limitation for CubeSats makes that solution undesirable. Unlike traditional dual spinners, which use the spinning part of the spacecraft to stabilize the pointing, for MicroMAS-1, the dual-spinner compensates for the payload spin. This solution offers the relative simplicity of a three-axis stabilized design. While the long-axis reaction wheel will suffer reduced control authority (since its equilibrium spin rate is nonzero to counter the spinning payload), few disturbance torques affect the satellite about its long axis, which is aligned with the spacecraft's velocity vector.

The design process for MicroMAS-1 involved an initial trade study on overall ADCS architectures (resulting in the three-axis stabilized dual-spinner) and several trade studies on COTS ADCS solutions (resulting in the selection of the MAI-400 mini-ADACS system), as well as extensive six-degree-of-freedom modeling of the spacecraft, sensor, and actuator dynamics for estimator and controller development.

Testing and verification of the ADCS is imperative since operation of the payload and communications systems depend upon the $2 \mathrm{U}$ bus remaining fixed with the communications antenna nadir-pointing while the payload rotates. The currently selected ADCS assembly is from Maryland Aerospace Inc., and contains a triaxial fluxgate magnetometer, two infrared Earth Horizon Sensors, and three torque rods, in addition to the reaction wheels ${ }^{18}$. The reaction wheels serve as the primary means of canceling the momentum from the rotating payload, precessing the spacecraft as it orbits, and cancelling disturbance torques. 


\section{Power}

The orientation requirements for MicroMAS-1 do not permit sun tracking for maximum power generation. This drove the decision to use four 3U double-sided deployable solar panels oriented at $90^{\circ}$ angles to the structure. Another $2 \mathrm{U}$ solar panel will be body-mounted on the zenith side of the satellite bus to provide additional power, as seen in Figure 7. In Figure 7, the color blue indicates the areas that will be populated with solar cells. This configuration has been modeled to generate an average power of $20.0 \mathrm{~W}$ in sunlight $(12.7 \mathrm{~W}$ orbit average) at a $600 \mathrm{~km}$ altitude orbit when UTJ cells (28.5\% efficiency) are used. MicroMAS-1 power subsystem components are produced by Clyde Space ${ }^{19}$.

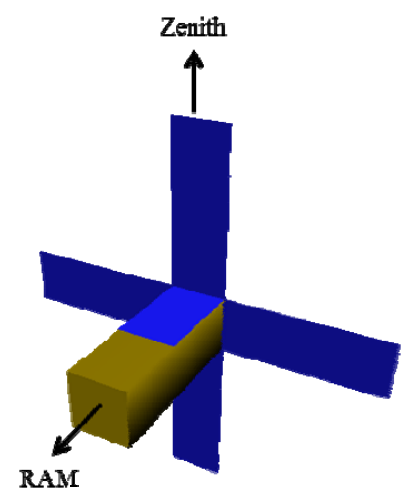

Figure 7: MicroMAS-1 Solar Array Configuration

The EPS conducts power monitoring and conditioning and adjusts the operational voltage and current of photovoltaic cells to maximize power generation. The power is then stored in rechargeable $30 \mathrm{~W}$-hr lithium polymer batteries or sent directly to the loads at voltage levels of 3.3 , 5 , or $12 \mathrm{~V}$.

Table 1 shows a high-level power budget for the mission. The components included in each subsystem are:

- $\quad$ ADCS: MAI-400, Magnetometer, MEMS gyro

- Avionics: Motherboard-Level Shifter, Motherboard-AND Gate, Pluggable Processor Module

- Communications: Modem (transmitting)

- Payload: Radiometer

- $\quad$ Power: EPS, Battery Charging

- Structures: Motor, Motor Controller, Encoder, Thermal Management

The peak power value takes into account losses from converter inefficiencies. All subsystems will be nominally functioning all the time except for the transceiver. The effective duty cycle for the transceiver assumes that MicroMAS-1 is transmitting for ten minutes out of each 64 minutes of daylight, is on standby for one minute, and is off for the remaining 53 minutes.
The average generated power currently has a margin of only $7 \%$ over the current expected average required power. This is a low margin to have, and a plan to handle potential power deficiency is being investigated. As flight components are being procured and tested, the power budget will be updated. Once all of the subsystem component specifications are confirmed, we will have a true indication of whether or not MicroMAS-1 can remain power positive. In the case that MicroMAS-1 cannot remain power positive, a payload duty cycle will be implemented.

\section{Table 1: Power Budget (BOL Daylight)}

\begin{tabular}{|l|c|c|}
\hline \multicolumn{1}{|c|}{ Subsystem } & $\begin{array}{c}\text { Peak Power } \\
\text { Consumption (W) }\end{array}$ & $\begin{array}{c}\text { Effective } \\
\text { Duty Cycle }\end{array}$ \\
\hline ADCS & 3.4 & 1 \\
\hline Avionics & 0.3 & 1 \\
\hline Communications & 9.0 & 0.16 \\
\hline Payload & 3.3 & 1 \\
\hline Power & 7.5 & 1 \\
\hline Structures & 2.3 & 1 \\
\hline Average Consumed Power & \multicolumn{2}{|c|}{18.7} \\
\hline Average Generated Power & \multicolumn{2}{|c|}{$7.1 \%$} \\
\hline Power Margin & \multicolumn{2}{|c|}{} \\
\hline
\end{tabular}

\section{Data Management}

The onboard communications system consists of an Sband transceiver and wide-beam width patch antenna. The flight transceiver is the Espace Payload Telemetry System (PTS). The PTS supports S-band communications with cyclic-redundancy checking, fits in a CubeSat form factor, and provides up to $1 \mathrm{~W}$ of transmission power output. MicroMAS-1 will use the OSAGS for uplink of commands and downlink of data. OSAGS consists of ground stations at Kwajalein Island, Cayenne, and Singapore. Uplink frequencies are between 2.0252.120 GHz and downlink frequencies are between 2.200$2.300 \mathrm{GHz}$ at up to $3.5 \mathrm{Mbps}$. Current estimates of payload data production indicate that the onboard communications systems will downlink data at a peak rate of approximately $230 \mathrm{kbps}$.

For command and data handling (C\&DH), MicroMAS-1 will use a Pumpkin CubeSat Motherboard with a Microchip PIC24 microcontroller as the flight computer. MicroMAS-1 uses Pumpkin's Salvo Real Time Operating System, and each peripheral (magnetometer, gyroscope, reaction wheels, modem, motor controller, payload, SD card) of the satellite will run as an operating system task in Salvo ${ }^{20}$. Physical interfaces to the MAI401 reaction wheels, modem, and SD card will be via the 
CubeSat Kit bus, while interfaces to the magnetometer, gyroscope, motor controller, and payload will be through custom-designed PCBs.

\section{TEST RESULTS}

MicroMAS-1 went through a design iteration with an EDM build during the Summer 2011 to test the areas of greatest risk faced by the mission, namely the spinner assembly and the ADCS. Six main tests were completed on the EDM: System Integration, Spinner Assembly Data Interface, Solar Array Orientation, Vibration Testing, Thermal Testing, and a Power Cycling Test. This section summarizes the results of the testing done on the EDM and analysis done for the flight model.

In order to conduct thermal-vacuum and vibration testing of the EDM without risking damage to expensive components, a structural model that mimicked the SWaP output of the EDM was created. The model consisted of a $2 \mathrm{U}$ skeleton chassis manufactured by Pumpkin, Inc; aluminum plates with identical shape and mass properties to their functional counterparts; power resistors to dissipate heat loads; and a mock spinner assembly using a COTS bearing, COTS gears, and motor.

\section{Vibration Testing}

Vibration testing of the SWAP model was conducted at MIT Lincoln Laboratory from 24-31 August 2011. The following testing profile was conducted three times once each for the $\mathrm{X}$-, $\mathrm{Y}$-, and $\mathrm{Z}$-axis.

- Pre-test white noise random signature test from $20-2000 \mathrm{~Hz}$ at a level of $0.5 \mathrm{G} \_r m s$ for 60 seconds (conducted during pre-test set-up).

- $\quad-12 \mathrm{~dB},-6 \mathrm{~dB}$, and full-level (20 G_rms) sine burst tests at $50 \mathrm{~Hz}$ for 1.6 seconds.

- $\quad$ Post-test white noise random signature.

- $\quad-12 \mathrm{~dB},-6 \mathrm{~dB}$, and full-level (14.1 G_rms) random tests for 60 seconds.

- $\quad$ Post-test white noise random signature test from $20-2000 \mathrm{~Hz}$ at a level of $0.5 \mathrm{Grms}$ for 60 seconds.

A summary of the major (Y-axis) modes of the MicroMAS-1 SWAP model is provided in Table 2. Four of the first five modes were caused by the solar panels, with the stowed deployable panels being the largest contributor. This was attributed to the method used to restrain the deployable panels. Two sets of nylon tiedowns were used to restrain the panels. The inner set was located in the middle of the panel, securing the panels directly to the chassis. The second set was placed near the tip of the panels, running through the center of the payload cube. At this location, the panels were secured to each other, rather than to a rigid structure. This gave the tips of the panels a certain degree of freedom to deflect. For the FM will use a tie-down method as designed by Clyde Space between the deployable and body-mounted panels will be performed to ensure that they minimize deflection. Additionally, ends of the solar panels may be tied to the payload should any modes be generated after implementation of the Clyde Space tiedown method.

The remaining (second) mode was caused by the MAI201 mini-ADACS unit, which underscored some preexisting concerns with the mounting scheme. At the end of Y-Axis testing, it was discovered that two of the four internal mounting rails had failed; they sheared right above the nuts securing the top ADCS mounting plate. It is unknown at which point during the test profile this failure occurred, but it is likely that this was the cause of the $43 \mathrm{~Hz}$ first-mode frequency shift between the beginning (194 Hz@28 G/G seen during baseline white noise) and end (151 Hz @ 12.5 G/G seen during postrandom white noise) of testing.

Table 2: Y-Axis Modes of MicroMAS SWAP model

\begin{tabular}{|c|c|c|c|}
\hline Mode & Location & $\begin{array}{c}\text { Frequency } \\
\text { (Hz) }\end{array}$ & $\begin{array}{c}\text { Amplification } \\
\text { (Q) }\end{array}$ \\
\hline First & $\begin{array}{l}\text { Deployable Solar } \\
\text { Panel }\end{array}$ & 97.5 & 6.15 \\
\hline Second & MAI-201 ADCS & 151 & 12.5 \\
\hline Third & $\begin{array}{l}\text { Deployable Solar } \\
\text { Panel }\end{array}$ & 234 & 18.1 \\
\hline Fourth & 2U Solar Panel & 286 & 12.8 \\
\hline Fifth & $\begin{array}{l}\text { Deployable Solar } \\
\text { Panel }\end{array}$ & 475 & 56.6 \\
\hline
\end{tabular}

The results of this test informed two major areas of the FM design. The first change related to the mounting of the internal components. The $20 \mathrm{~cm}$-long rails that ran the entire length of the $2 \mathrm{U}$ chassis were replaced with sets of male-female threaded standoffs between each component board. This resulted in increased structural rigidity of the bus stack.

The second change was related to the mounting of the ADCS unit. For the flight model, the MAI-400 miniADACS unit is being used instead of the MAI-201. The MAI-400 offers the increased structural rigidity as it mounts securely to the bottom of the CubeSat chassis and is further constrained during launch by the P-POD rails. The size of the MAI-400 (0.5U) makes this relatively easy to accommodate. This is opposed to the MAI201 which although smaller, was more difficult to mount and achieve structural rigidity within the EDM bus. 


\section{Thermal Testing}

Thermal-vacuum testing was conducted on the MicroMAS-1 SWAP model at the MIT Kavli Institute vacuum chamber during the week of August 8-12, 2011. The purpose of the test was twofold. The first objective was to simulate on-orbit conditions and establish approximate heating and cooling transients for the satellite. The second objective was to generate a set of data under known conditions against which we could compare and verify our thermal model.

Table 3 shows six major tests that were performed and the resulting thermal observations. The most critical anomaly occurred after four days in operation under vacuum. The COTS motor failed. From post-test analysis of the spinner assembly, we concluded that outgassing of the lubricant in the bearing, leading to increased friction, was the potential reason for the motor failure. The spinner assembly did, however, still operate in standard temperature and pressure (STP) conditions, albeit with additional noise and resistance. It should be noted that the motor used in this configuration was not rated for space, and that out-gassing of the lubricants was likely to happen. To address this issue for the FM, we are using a space-rated a zero-cogging brushless DC motor. The newly selected motor has been tested by the vendor in relevant environments, and meets the temperature and vacuum requirements.

Table 3: Thermal-Vacuum Test Case Definitions

\begin{tabular}{|l|l|l|l|}
\hline Test Case & SWAPSat & \multicolumn{1}{|c|}{ Chamber } & \multicolumn{1}{c|}{ Results } \\
\hline $\begin{array}{l}\text { T1-2 } \\
\text { Hot Case }\end{array}$ & On & Cold $\rightarrow$ Hot & Batteries ran hot \\
\hline $\begin{array}{l}\text { T1-3 } \\
\text { Power Off }\end{array}$ & On $\rightarrow$ Off & Hot & $\begin{array}{l}\text { All components } \\
\text { within operation- } \\
\text { al temperatures }\end{array}$ \\
\hline $\begin{array}{l}\text { T1-4 } \\
\text { Hot Start }\end{array}$ & Off $\rightarrow$ On & Hot & $\begin{array}{l}\text { All components } \\
\text { within operation- } \\
\text { al temperatures }\end{array}$ \\
\hline $\begin{array}{l}\text { T1-5 } \\
\text { Simult }\end{array}$ & On & Hot $\rightarrow$ Cold (x3) & $\begin{array}{l}\text { All components } \\
\text { within operation- } \\
\text { al temperatures }\end{array}$ \\
\hline $\begin{array}{l}\text { T1-6 } \\
\text { Cold Soak }\end{array}$ & On $\rightarrow$ Off & Cold & $\begin{array}{l}\text { Systems ap- } \\
\text { proached cold } \\
\text { limits }\end{array}$ \\
\hline $\begin{array}{l}\text { T1-7 } \\
\text { Cold Start }\end{array}$ & Off $\rightarrow$ On & Cold & $\begin{array}{l}\text { All components } \\
\text { within operation- } \\
\text { al temperatures }\end{array}$ \\
\hline
\end{tabular}

The thermal-vacuum test results were used to validate the COMSOL thermal model of MicroMAS-1. Through extensive testing, the thermal tape was found to have an effective emissivity of $0.55+/-0.05$. The bus heat load from avionics was found to be approximately $10.5 \mathrm{~W}$. These values were then used in the on-orbit thermal model. In a variety of on-orbit scenarios, MicroMAS-1 was determined to have an operating bus temperature of between -10 and $0{ }^{\circ} \mathrm{C}$. Preliminary thermal modeling in COMSOL shows that the satellite will run $5{ }^{\circ} \mathrm{C}$ colder than was indicated in the thermal vacuum test. This is due to the radiative surface of the deployed solar panels which were not included in the SWaP model. The only component this affects is the batteries, which are the most sensitive to cold temperatures and will have an internal heater.

\section{Avionics Demonstration}

To demonstrate functionality of the avionics subsystem, the data interfaces from avionics to each of the peripheral devices were tested in the EDM. All interfaces present at the time of the Engineering Design functioned correctly.

\section{ADCS Simulations}

For preliminary analysis, a six-degree-of-freedom (6DoF) simulation of the spacecraft's attitude and translational dynamics was created in MATLAB Simulink. The ADCS simulation includes disturbances due to gravity gradient, spherical gravitational harmonics using the EGM 2008 model, drag using the NRLMSISE-00 atmospheric density model, solar pressure, magnetospheric effects using the IGRF-11 model, and time-variable masses and inertia tensors (such as due to the deployment of the solar panels). Saturation, quantization, and noise have been modeled for the rate gyros, magnetometer, reaction wheels, and magnetorquers. These models will be updated as each of the components is further characterized.

Work on estimator and controller design is ongoing as the simulation is refined, and hardware-in-the-loop testing has begun on an ADCS testbed at MIT SSL. The ADCS testbed currently consists of a 3-DoF air bearing, an MAI-201 reaction wheel set, a prototyping avionics board, an MHX2420 modem for communication with a simulated ground station, and a battery of 16 AA cells. The avionics board contains a PIC24 microcontroller similar to the flight model, several voltage converters required to run the various components, and a rate gyro for initial feedback tests. Expansion of the testbed will include integration of a magnetometer to provide attitude data using the Helmholtz coil surrounding the air bearing, an accelerometer to mimic the MAI-400's Earth limb sensors' provision of a nadir vector (in this case, in the form of a gravitational acceleration vector), and ultimately the integration of the MAI-400 mini-ADACS system. The study will also include interaction of the attitude control and payload spinner control. This will consist of adding an active representation of the spinner assembly to provide representative interactions for the test. 


\section{Future Testing}

Development of the FM is currently underway, along with plans for more thorough testing and analysis in advance of the MicroMAS-1 critical design review (CDR). Future ADCS testing plans include mounting the FM spacecraft on the 3-DoF air bearing for final estimator and controller verification. Full-cycle power tests will confirm that the satellite will generate enough power to support a continuously-operating payload during the mission.

\section{SUMMARY}

MicroMAS-1 will demonstrate the feasibility of achieving science-quality radiometric data with improved revisit times and improved ground sampling distance using a CubeSat platform. Key payload technology developments include an ultra-compact intermediate frequency processor module for channelization, detection, and Ato-D conversion, a highly miniaturized payload antenna system, a highly integrated and power-optimized RF front-end electronics. Spacecraft bus developments include a unique dual-spinning $3 \mathrm{U}$ CubeSat design, design of the spinner assembly, and S-band communications boards. MicroMAS-1 is working toward a launch via the NASA ELaNa program in 2014.

\section{Acknowledgements}

The MicroMAS-1 development project is a collaboration between MIT Lincoln Laboratory and MIT SSL, as well as the MIT Earth and Planetary Sciences department. Graduate study has been funded in part by the MIT AeroAstro Department, the MIT SSL, and MIT Lincoln Laboratory. Undergraduate study has been funded in part by the Massachusetts Space Grant and by the MIT Undergraduate Research Opportunities Program.

The authors would like to acknowledge the work of all current and previous students on the MicroMAS-1 program. Additionally, the authors would like to acknowledge the invaluable advice of Paul Bauer.

\section{References}

1. Tomiyasu, K. "Remote Sensing of the Earth by Microwaves." Proceedings of the IEEE, vol. 62, no. 1, January 1974: 86-92.

2. Njoku, E. "Passive Microwave Remote Sensing of the Earth from Space - A Review." Proceedings from the IEEE, vol. 70, no. 7, 1982: 728-750.

3. Lambrigsten, B., S.T. Brown, T. C. Gaier, L. Herrell, P. P. Kangaslahti, and A.B. Tanner. "Monitoring the Hydrologic Cycle with the PATH Mission." Proceedings of the IEEE, vol. 98, no. 5, 2010: 862-877.
4. Katsaros, K. B., P. W. Vachon, W. T. Liu, and P. G. Black. "Microwave Remote Sensing of Tropical Cyclones from Space." Journal of Oceanography, vol. 58, 2002: 137-151

5. Surussavadee, C., and D. Staelin, "NPOESS Precipitation Retrievals Using the ATMS Passive Microwave Spectrometer," IEEE Geoscience and Remote Sensing Letters, vol. 7, no. 3, July 2010: 440-444.

6. Bell, W., S. English, B. Candy, N. Atkinson, F. Hilton, N. Baker, S. Swadley, W. Campbell, N. Bormann, G. Kelly, and M. Kazumori, "The Assimilation of SSMIS Radiances in Numerical Weather Prediction Models.” IEEE Transactions on Geoscience and Remote Sensing, vol. 46, no. 4, April 2008: 884-900.

7. Spina, M. S., M. J. Schwartz, D. H. Staelin, and A. J. Gasiewski. "Application of Multilayer Feedforward Neural Networks to Precipitation Cell-Top Altitude Estimation." IEEE Transactions on Geoscience and Remote Sensing, vol. 36, no. 1, 1998: 154-162.

8. Ball Aerospace. "NPP Media Kit." Ball Aerospace and Technologies Corporation, 2012: http://www.ballaerospace.com/page.jsp?page $=254$

9. United States General Accounting Office. Letter to the Subcommittee on National Security Committee on Appropriations, House of Representatives. February 6, 1995. Meteorological Satellites, GAO/NSIAD-95-87R

10. United States Government Accountability Office, "Polar-Orbiting Satellites: With Costs Increasing and Data Continuity at Risk, Improvements Needed in Tri-Agency Decision Making.” Testimony before the Subcommittee on Investigations and Oversight, Committee on Science and Technology, House of Representatives. GAO-09-772T, June 17, 2009

11. Blackwell, W. J., et al. "Hyperspectral Microwave Atmospheric Sounder." IEEE Transactions on Geoscience and Remote Sensing, vol. 49, no. 1, 2011: 128-142.

12. Kangaslahti, P. "Recent developments in $180 \mathrm{GHz}$ MMIC LNA and receiver technology." 11th Specialist Meeting on Microwave Radiometry and Remote Sensing of the Environment, 2010: 272275. 
13. Brown, S. T., S. Desai, W. Lu, and A. Tanner. "On the Long-Term Stablilty of Microwave Radiometers Using Noise Diodes for Calibration." IEEE Transactions on Geoscience and Remote Sensing, vol. 45, no. 7, July 2007: 1908-1920.

14. National Aeronautic and Space Administration. "Announcement of CubeSat Launch Initiative." August 5, $2011 . \quad$ Posted: http://prod.nais.nasa.gov/cgibin/eps/bizops.cgi?gr=D\&pin=04\#147535

15. National Aeronautic and Space Administration, "Process for Limiting Orbital Debris." NASA Technical Standard NASA-STD-8719.14A, Dec. 2008

16. Pumpkin, Incorporated. "3D Models of the CubeSat Kit." 2011. <http://www.cubesatkit.com/content/design.html>.

17. The CubeSat Program, CalPoly SLO. "CubeSat Design Specifications.” 2009. http://cubesat.org/images/developers/cds_rev12.pd f.

18. Maryland Aerospace. The 1/2U MAI-400 A La Carte. 2011.

$<$ http://www.miniadacs.com/linked/2012-0427\%20mai400\%20a\%20la\%20carte\%20specification.pdf>.

19. Clyde Space. CubeSat Shop. 2012. <http://www.clyde-space.com/cubesat_shop>.

20. Pumpkin, Incorporated. "Available Datasheets." 2011.

$<$ http://www.cubesatkit.com/content/datasheet.htm $\mathrm{l}>$. 